Meta

Journal des traducteurs

Translators' Journal

\title{
Lexique anglais-français de la facture de clavecins
}

\section{Louise Brunette}

Volume 38, numéro 2, juin 1993

URI : https://id.erudit.org/iderudit/002158ar

DOI : https://doi.org/10.7202/002158ar

Aller au sommaire du numéro

Éditeur(s)

Les Presses de l'Université de Montréal

ISSN

0026-0452 (imprimé)

1492-1421 (numérique)

Découvrir la revue

Citer cet article

Brunette, L. (1993). Lexique anglais-français de la facture de clavecins. Meta, 38(2), 332-344. https://doi.org/10.7202/002158ar d'utilisation que vous pouvez consulter en ligne.

https://apropos.erudit.org/fr/usagers/politique-dutilisation/ 


\section{LEXIQUE ANGLAIS-FRANÇAIS DE LA FACTURE DE CLAVECINS}

Depuis une trentaine d'années, le vocabulaire anglais du clavecin, et en particulier celui de la facture de l'instrument, a été largement inventorié et étudié par les historiens américains de la facture. Quant à la terminologie originale française du domaine, elle n'a subi aucune révision exhaustive depuis l'effort de systématisation de L'Encyclopédie. Il n'existe pas non plus d'ouvrages sérieux de terminologie comparée mettant à la disposition des traducteurs (anglais-français) des équivalents sûrs. Ce petit lexique bilingue de la facture de clavecins voudrait combler en partie cette lacune. 
Les quelque 200 notions présentées ici sous forme de lexique bilingue appartiennent principalement aux six sous-domaines suivants : mécanique de l'instrument, production du son, caisse, décoration, instruments apparentés et histoire. On trouvera également quelques termes relatifs à l'entretien et à l'accord de l'instrument.

Les termes anglais et français donnés en entrée sont toujours les expressions les plus souvent relevées dans les sources dépouillées ou dans le vocabulaire des experts interrogés. Ils sont suivis des synonymes, apparaissant aussi par ordre de fréquence, s'il y a lieu.

Il est à espérer que le renouveau de la musique ancienne inspire d'autres recherches du genre de celle-ci : terminologues, traducteurs, musiciens et constructeurs d'instruments $\mathrm{y}$ trouveront tous leur bénéfice.

\begin{tabular}{|c|c|}
\hline a tempera & $\begin{array}{l}\text { à la détrempe } \\
\text { a tempera }\end{array}$ \\
\hline action & mécanique \\
\hline $\begin{array}{l}\text { adjustment screw } \\
\text { voicing screw }\end{array}$ & vis de réglage \\
\hline arcade & arcade \\
\hline $\begin{array}{l}\text { attack, to } \\
\text { strike, to }\end{array}$ & $\begin{array}{l}\text { frapper } \\
\text { percuter }\end{array}$ \\
\hline $\begin{array}{l}\text { axle pin } \\
\text { pivot }\end{array}$ & axe \\
\hline $\begin{array}{l}\text { back eight foot } \\
\text { lower eight foot }\end{array}$ & $\begin{array}{l}\text { huit pieds principal } \\
\text { principal }\end{array}$ \\
\hline back rail & $\begin{array}{l}\text { barre de repos } \\
\text { traverse arrière }\end{array}$ \\
\hline $\begin{array}{l}\text { balance pin } \\
\text { balance rail pin }\end{array}$ & $\begin{array}{l}\text { pointe de balancier } \\
\text { pivot }\end{array}$ \\
\hline balance point & $\begin{array}{l}\text { point de balance } \\
\text { point de bascule }\end{array}$ \\
\hline balance rail & $\begin{array}{l}\text { balancier } \\
\text { barre de balancier, } \\
\text { traverse de pivots }\end{array}$ \\
\hline $\begin{array}{l}\text { band } \\
\text { banding, stringing }\end{array}$ & filet \\
\hline $\begin{array}{l}\text { bass } \\
\text { bass side, bass end }\end{array}$ & $\begin{array}{l}\text { grave } \\
\text { extrémité grave }\end{array}$ \\
\hline beat, $n$ & battement \\
\hline $\begin{array}{l}\text { belly rail } \\
\text { bellyrail, header, } \\
\text { counter wrestplank }\end{array}$ & $\begin{array}{l}\text { contre-sommier } \\
\text { masse supérieure }\end{array}$ \\
\hline bentside & $\begin{array}{l}\text { courbe } \\
\text { éclisse courbe, } \\
\text { éclisse concave }\end{array}$ \\
\hline
\end{tabular}


block printed paper printed paper

Boston School

bottom

box slide

boxslide, box guide

brace

gap spacerbar

breadboard end

bridge

bridge pin

bristle

jack spring

broken octave

split-sharp short octave

buff batten

buff stop

harp stop

cap moulding

carrying case

transportation cover

case

case side

cheek

cheekpiece

chinoiserie decoration

choir (of strings)

set of strings

clavecin brisé

clavichord

cloth punching

balance punching

compass

range papier imprimé

école de Boston

fond

guide à l'italienne

étrésillon

étançon

alèse

alaise

chevalet

pointe de chevalet

pointe

ressort

ressort de languette

octave brisée

octave courte avec feintes brisées

réglette du jeu de buffle

sourdine

jeu de luth

moulure de caisse

moulure supérieure

housse de transport

caisse

coffre

éclisse

côté

joue

chinoiserie

chcur

rang de cordes

clavecin brisé

clavicorde

mouche

étendue 
couple, to

coupler

keyboard coupler

mechanism,

manual coupler

coupler dog

dog

cutoff bar

cut-off bar

cyma

ogee

damper

decoration

delrin

delrin plastic

disposition

dogleg

dogleg

double bentside

double curve bentside,

double-curved bentside

double harpsichord

double-manual

harpsichord

double tongued jack

double jack

dovetailed

drawstop

handstop (1),

push-pull stop

eight foot

eight foot stop, unison

equal temperament

escapement (I)

escapement (2)

four-foot hitchpin rail boudin accoupler les claviers

accouplement

pilote

pilote d'accouplement

grande barre

grande barre diagonale,

diagonale

doucine

étouffoir

décoration

décor

delrin

Delrin

disposition

dogleg jack

dog-leg

éclisse à double courbe

clavécin à double clavier

clavecin à deux claviers

sautereau à double languette

(monté) en queue d'aronde

tirette

tirette de registre,

canne

huit pieds

jeu de huit pieds

tempérament égal

renvoi de la languette

mouvement de bascule

contre-chevalet

boudin 


\begin{tabular}{|c|c|}
\hline $\begin{array}{l}\text { four-foot stop } \\
\text { four foot stop, } \\
\text { four foot register }\end{array}$ & $\begin{array}{l}\text { quatre pieds } \\
\text { jeu de quatre pieds }\end{array}$ \\
\hline fourth & quarte \\
\hline $\begin{array}{l}\text { frame } \\
\text { framing, bracing }\end{array}$ & $\begin{array}{l}\text { charpente } \\
\text { traverses }\end{array}$ \\
\hline fretted clavichord & clavicorde lié \\
\hline $\begin{array}{l}\text { front eight foot } \\
\text { upper manual eight foot, } \\
\text { upper eight foot }\end{array}$ & $\begin{array}{l}\text { second jeu de huit pieds } \\
\text { second huit pieds }\end{array}$ \\
\hline front rail & traverse avant \\
\hline gap & fosse \\
\hline Guild of Saint Luke & guilde de Saint-Luc \\
\hline $\begin{array}{l}\text { handstop (2) } \\
\text { hand stop }\end{array}$ & manette \\
\hline harpsichord & clavecin \\
\hline $\begin{array}{l}\text { harpsichord maker } \\
\text { harpsichord builder }\end{array}$ & facteur de clavecins \\
\hline $\begin{array}{l}\text { harpsichord making } \\
\text { harpsichord building }\end{array}$ & facture (de clavecins) \\
\hline historical harpsichord & $\begin{array}{l}\text { clavecin de facture classique } \\
\text { clavecin de facture ancienne, } \\
\text { clavecin historique, } \\
\text { clavecin de facture historique }\end{array}$ \\
\hline $\begin{array}{l}\text { hitchpin } \\
\text { hitch-pin }\end{array}$ & $\begin{array}{l}\text { pointe d'accroche } \\
\text { pointe d'attache }\end{array}$ \\
\hline hitchpin rail & $\begin{array}{l}\text { cordier } \\
\text { moulure d'accroche }\end{array}$ \\
\hline hog's bristle & soie de porc \\
\hline inside of lid & $\begin{array}{l}\text { dessous de couvercle } \\
\text { intérieur de couvercle }\end{array}$ \\
\hline ivory stud & bouton d'ivoire \\
\hline jack & sautereau \\
\hline $\begin{array}{l}\text { jackrail } \\
\text { jack rail }\end{array}$ & chapiteau \\
\hline just scale & $\begin{array}{l}\text { juste proportion } \\
\text { règle de la juste } \\
\text { proportion }\end{array}$ \\
\hline $\begin{array}{l}\text { keyboard } \\
\text { manual }\end{array}$ & clavier \\
\hline
\end{tabular}


key block

end block, side block

key dip

key frame

key bed, keyframe

keyframe side

key front

key head

key lever (I)

key lever (2)

key panel

keyboard panel

key tail

key top

keywell

kit

kit harpsichord

knee lever

lid flap

lid stick

lidstick

liner

lockboard

lock strip

lower belly rail

lower guide

jack guide

lower level frame

lower frame, lower brace

lower manual

lower keyboard

lute stop

machine stop

machine

marbling

marquetry bloc

bloc de clavier

enfoncement de la touche

châssis de clavier

bras de châssis

traverse latérale

fronton

avant de la marche

touche

levier

panneau des touches

queue de palette

palette

placage

boîte de clavier

kit

genouillère

abattant

béquille

canne

contre-éclisse

portillon

masse

guide inférieur

guide inférieur fixe

traverse de fond

traverse inférieure, traverse

clavier inférieur

nasal

jeu nasal, jeu de nasal

machine stop

machine-stop

faux marbre

marqueterie 


\begin{tabular}{|c|c|}
\hline $\begin{array}{l}\text { mortise } \\
\text { mortice }\end{array}$ & mortaise \\
\hline motto & devise \\
\hline $\begin{array}{l}\text { moulding } \\
\text { molding }\end{array}$ & moulure \\
\hline music desk & pupitre \\
\hline music wire & $\begin{array}{l}\text { corde de métal } \\
\text { corde métallique }\end{array}$ \\
\hline name batten & $\begin{array}{l}\text { barre d'adresse } \\
\text { listel }\end{array}$ \\
\hline $\begin{array}{l}\text { nameboard } \\
\text { frontboard }\end{array}$ & $\begin{array}{l}\text { gorge } \\
\text { tour de clavier, devant }\end{array}$ \\
\hline $\begin{array}{l}\text { natural } \\
\text { key natural } \\
\text { note diatonique }\end{array}$ & $\begin{array}{l}\text { marche } \\
\text { touche diatonique, }\end{array}$ \\
\hline normal pitch & diapason normal \\
\hline $\begin{array}{l}\text { nut } \\
\text { wrest plank bridge }\end{array}$ & sillet \\
\hline nut pin & pointe (de sillet) \\
\hline octave span & mesure de l'octave \\
\hline off, adj. & hors jeu \\
\hline on, adj. & en jeu \\
\hline outer case & caisse extérieure \\
\hline $\begin{array}{l}\text { overrail } \\
\text { touchrail }\end{array}$ & barre d'arrêt \\
\hline $\begin{array}{l}\text { overspun string } \\
\text { covered string }\end{array}$ & corde filée \\
\hline padding & habillage \\
\hline $\begin{array}{l}\text { peau de buffle (l) } \\
\text { peau de buffle stop }\end{array}$ & jeu de buffle \\
\hline peau de buffle (2) & peau de buffle \\
\hline pedal & pédale \\
\hline pedals & pédalier \\
\hline plectrum & $\begin{array}{l}\text { plectre } \\
\text { bec }\end{array}$ \\
\hline pluck, to & pincer \\
\hline plucked instrument & instrument à cordes pincées \\
\hline plucking mechanism & mécanisme pincé \\
\hline
\end{tabular}




\begin{tabular}{|c|c|}
\hline plucking order & ordre de pincement \\
\hline $\begin{array}{l}\text { plucking point } \\
\text { point of pluck }\end{array}$ & $\begin{array}{l}\text { point de pincement } \\
\text { point d'attaque }\end{array}$ \\
\hline projecting slide & guide à la flamande \\
\hline $\begin{array}{l}\text { quarter, on the } \\
\text { quartesawed, quartered }\end{array}$ & (taillé) sur quartier \\
\hline quill, $\mathrm{n}$. & plume \\
\hline quill, to & emplumer \\
\hline quilled jack & sautereau emplumé \\
\hline quilling & emplumage \\
\hline $\begin{array}{l}\text { rack } \\
\text { diapason }\end{array}$ & $\begin{array}{l}\text { peigne } \\
\text { diapason }\end{array}$ \\
\hline rack pin & $\begin{array}{l}\text { pointe du guide } \\
\text { flipot }\end{array}$ \\
\hline ravalement & ravalement \\
\hline $\begin{array}{l}\text { rear of key } \\
\text { end of key }\end{array}$ & queue de touche \\
\hline $\begin{array}{l}\text { register } \\
\text { jackslide, slide, } \\
\text { upper guide }\end{array}$ & $\begin{array}{l}\text { registre } \\
\text { guide supérieur }\end{array}$ \\
\hline registration & registration \\
\hline recessed keyboard & $\begin{array}{l}\text { clavier rentrant } \\
\text { clavier en retrait }\end{array}$ \\
\hline$r i b$ & $\begin{array}{l}\text { barre de table } \\
\text { barre }\end{array}$ \\
\hline $\begin{array}{l}\text { ribbing } \\
\text { soundboard ribbing }\end{array}$ & $\begin{array}{l}\text { barrage } \\
\text { barrage de table }\end{array}$ \\
\hline rose & $\begin{array}{l}\text { rose } \\
\text { rosace, rosette }\end{array}$ \\
\hline round notch & grain d'orge \\
\hline $\begin{array}{l}\text { row of jacks } \\
\text { set of jacks }\end{array}$ & rangée de sautereaux \\
\hline $\begin{array}{l}\text { scale } \\
\text { scaling }\end{array}$ & module (de cordes) \\
\hline scored line & trait gravé \\
\hline scroll & volute \\
\hline set in vibration, to & mettre en vibration \\
\hline
\end{tabular}




sharp
accidental
accidental key
short octave
shove coupler
push coupler
single manual
harpsichord
sixteen foot stop
sixteen foot,
sixteen foot register
soundboard
soundboard moulding
spacing
speak
speaking length
vibrating length
spine
spinet
split sharp
split accidental
stand
stop
stop lever
register lever,
register stop lever
stretcher
string, to
stringband
stringing ( 1 )
stringing ( $)$

feinte

touche chromatique, note chromatique

octave courte

accouplement en tiroir accouplement à tiroir

clavecin à un clavier

seize pieds

jeu de seize pieds

table d'harmonie

table

couvre-joint

espacement des touches

parler

longueur vibrante

longueur utile

échine

grande éclisse droite, grand côté

épinette

feinte brisée

piètement

pied

jeu

mouvement

entretoise

ceinture inférieure

mettre en corde

cordage

cordage

disposition des cordes

mise en corde

pointe

pan coupé

tangente 


\begin{tabular}{|c|c|}
\hline tongue & languette \\
\hline top of jack & tête de sautereau \\
\hline touch, $\mathrm{n}$. & toucher, n. m. \\
\hline transpose, to & transposer \\
\hline $\begin{array}{l}\text { transposing double } \\
\text { harpsichord } \\
\text { transposing harpsichord }\end{array}$ & clavecin transpositeur \\
\hline transposing instrument & instrument transpositeur \\
\hline transposition & transposition \\
\hline treble & aigu \\
\hline treble side & extrémité aiguë \\
\hline trestle stand & $\begin{array}{l}\text { piètement-tréteaux } \\
\text { piètement à tréteaux }\end{array}$ \\
\hline tuner & accordeur \\
\hline $\begin{array}{l}\text { tuning hammer } \\
\text { tuning key }\end{array}$ & $\begin{array}{l}\text { clef d'accord } \\
\text { accordoir }\end{array}$ \\
\hline $\begin{array}{l}\text { two foot } \\
\text { two foot stop }\end{array}$ & $\begin{array}{l}\text { deux pieds } \\
\text { jeu de deux pieds, } \\
\text { basse de } 2 \text { pieds }\end{array}$ \\
\hline unfretted clavichord & clavicorde libre \\
\hline $\begin{array}{l}\text { upper keyboard } \\
\text { upper manual }\end{array}$ & clavier supérieur \\
\hline $\begin{array}{l}\text { upper level frame } \\
\text { upper brace }\end{array}$ & $\begin{array}{l}\text { traverse supérieure } \\
\text { arc-boutant }\end{array}$ \\
\hline $\begin{array}{l}\text { upright harpsichord } \\
\text { clavicytherium }\end{array}$ & $\begin{array}{l}\text { clavecin vertical } \\
\text { clavicytherium }\end{array}$ \\
\hline veneer, $\mathrm{n}$. & placage \\
\hline Venitian swell & jalousie vénitienne \\
\hline virginal & $\begin{array}{l}\text { virginale } \\
\text { virginal }\end{array}$ \\
\hline voicing & harmonisation \\
\hline $\begin{array}{l}\text { wing-shaped spinet } \\
\text { bentside spinet, } \\
\text { leg-of-mutton }\end{array}$ & $\begin{array}{l}\text { épinette en aile d'oiseau } \\
\text { épinette en forme d'aile, } \\
\text { épinette à côté courbe }\end{array}$ \\
\hline $\begin{array}{l}\text { wolf }(1) \\
\text { wolf tone }(1) \\
\text { wolf interval }\end{array}$ & quinte du loup \\
\hline $\begin{array}{l}\text { wolf }(2) \\
\text { wolf note, wolf tone }(2)\end{array}$ & loup \\
\hline
\end{tabular}




\author{
wrest pin \\ wrestpin, tuning pin \\ wrest plank \\ pinblock \\ wrest plank block \\ support block, \\ thrust block, \\ wrestplank support block
}

cheville d'accord

cheville

sommier

renfort de sommier

renfort de paroi
LOUISE BRUNETTE Montréal, Canada

\section{BIBLIOGRAPHIE}

ABONDANCE, F. (1981): Restauration des instruments de musique, Fribourg, Suisse, Office du Livre.

ANSELM, Alain (1985): «Un clavecin singulier: Blanchet 1736», Musique ancienne, $\mathrm{n}^{\circ}$ 20, décembre 1985, Bourg-la-Reine, Cael, pp. 24-66.

APEL, Willi (1969): Harvard Dictionary of Music, 2nd edition revised and enlarged, Cambridge MA, The Belknap Press of Harvard University Press.

Encyclopédie méthodique. Arts et métiers mécaniques (1972): «Art du faiseur d'instruments de musique et lutherie», Genève, Minkoff Reprint.

BAINES, Anthony (dir) (1976): «Glossary of Technical and Acoustic Terms», Musical Instruments Through the Ages, New York Walker and Co., pp. 315-322.

BARNES, John (1987): «Annexe $\mathrm{N}^{\circ} 1$ Relative au clavecin $\mathrm{N}^{\circ} 1$ \%, Jean Tournay, Archives Dulcken, vol. 1 , Bruxelles, The Brussels Museum of Musical Instruments, «Bulletin», vol. XVII, $234 \mathrm{p}$.

BEDOS de CELLES, François (1963-1966): L'art du facteur d'orgues par Dom Bedos de Celles, FaksimileNachdruck hrsg. von Christhard Mohrenholz, Kassel, Bärenreiter, 4 t. en 3 vol., vol. 1 reproduction de Band I: 1766; vol. 2 de Band II / III : 1770 ; vol. 3 de Band IV : 1778 .

BINETTE, Reynald (1985) : Vocabulaire du tournage du bois, Québec, Gouvernement du Québec, Cahiers de l'Office de la langue française, Terminologie technique et industrielle, $39 \mathrm{p}$.

BRAGARD, R. et Ferd. J. DE HEN (1967): Les instruments de musique dans l'art et l'histoire, RhodeSt-Genèse, Belgique, Albert de Visscher Éditeur, $258 \mathrm{p}$.

BRENET, Michel (1926): Dictionnaire pratique et historique de la musique, Paris, Librairie Armand Colin, $487 \mathrm{p}$.

CANDE, Roland de (1961): Dictionnaire de musique, Paris, Seuil, 284 p. (Microcosme, 1).

[Catalogue] Von Nagel Paris (1991) : Paris, Ateliers Von Nagel, 38 p. + supplément anglais.

Chambers Science and Technology Dictionary (1988): New York, W. \& R. Chambers Ltd and Cambridge University Press, $1008 \mathrm{p}$.

CLOSSON, Ernest et Charles VAN DER BORREN (dir.) (1950): La musique en Belgique, Bruxelles, La Renaissance du livre, $486 \mathrm{p}$.

CORNELOUP, Marcel (1965?): L'orchestre et ses instruments: initiation musicale à travers les grandes auvres, [Paris], Presses d'Ile de France, $238 \mathrm{p}$.

DART, Thurston (1976): «The Clavichord», Anthony Baines (dir.), Musical Instruments Through the Ages, New York, Walker and Co., pp. 66-70.

DESCHAMPS, Jean (1987): «Annexe $\mathrm{N}^{\circ} 6$ Relative au clavecin No 9», Tournay, Jean, Archives Dulcken, vol. 1 , Bruxelles, The Brussels Museum of Musical Instruments, «Bulletin», vol. XVII, $234 \mathrm{p}$.

DIAGRAM GROUP (1976): Musical Instruments of the World, Paddington Press.

DOWD, William (1984): «The Surviving Instruments of the Blanchet Workshop», The Historical Harpsichord, vol. 1, Howard Schott (Ed.), [s. 1.], A monograph series in honor of Frank Hubbard, Pendragon Press, 125 p.

DUFOURCQ, Norbert (1981): Le clavecin, Paris, PUF, «Que sais-je ?», $\mathrm{n}^{\circ} 331,126 \mathrm{p}$.

DUMOULIN, Pierre (1977): «Le clavecin, son histoire, ses écoles», L'Europe du clavecin, Catalogue de l'exposition [tenue au] Palais Lascaris, Nice, juin-juillet 1977, Association des amis du Musée Instrumental du Conservatoire de région de Nice, pp. 25-96.

ELSEVIER (1956): Dictionnaire de cinéma, son et musique en six langues: anglais/américain, français, espagnol, italien, hollandais et allemand, préparé et classé selon l'ordre alphabétique par W. E. Clason, Paris, Dunod. 
MICHEL, François (dir.) (1958): Encyclopédie de la musique, en collaboration avec François Lesure et Vladimir Fedorov, Paris, Fasquelle, 3 vol.

Encyclopédie méthodique (1971): [v. 185-186], Framery, Nicolas Etienne, Musique/Compiled by Nicolas Etienne Framery et Pierre Louis Ginguené, New York, Capo Press, vol. 1, Réimpression de l'édition de 1791-1818.

The Golden Encyclopedia of Music (c1968): New York, Golden Press, 720 p.

Grand dictionnaire encyclopédique Larousse (1982): Paris, Librairie Larousse, 10 volumes.

SADIE, Stanley (Ed.) (1988): The Grove Concise Dictionary of Music, London, Macmillan Press, $850 \mathrm{p}$.

BLOM, Eric (Ed.) (1966): Grove's Dictionary of Music and Musicians, New York, St. Martin's Press Inc., volume IV.

HONEGGER, Marc (dir.) (1977): Dictionnaire de la musique; Science de la musique : Formes, Technique, Instruments, Paris, Bordas, 2 vol.

HUBBARD, Frank (1981): Le clavecin : trois siècles de facture, traduit par Hubert Bédard et Félia Bastet, Nogent-le-Roi, Jacques Laget, Éditions des Arts et métiers, 306 p., XLI pl.

HUBBARD, Frank (1973): I8th Century French Harpsichord in Kit Form. Instructions for Assembly, Decoration and Voicing, Waltham MA, 103 p. +23 pl.

HUBBARD, Frank (1988): Harpsichord Regulating and Repairing, Boston MA, Tuners Supply Inc., 48 p.

HUBBARD, Frank (1965): Three Centuries of Harpsichord Making, Cambridge MA, Harvard University Press, 373 p., XLI pl.

JURGENSON, William John Story (1991): Ruckers Enigma or Fifteen Years Search for the Unicorn, Lauffen, Allemagne, WJ, $18 \mathrm{p}$.

KOTTICK, Edward L. (1987): The Harpsichord Owner's Guide: A Manual for Buyers and Owners, Chapel Hill, University of North Carolina Press, $180 \mathrm{p}$.

Larousse de la musique (1982): Paris, Larousse, vol. 1.

LASKIN, William (1987): The World of Music Instruments Makers: A Guided Tour, Oakville, Canada, Mosaic Press, $109 \mathrm{p}$.

MAHILLON, Victor-Charles (1978): Catalogue descriptif et analytique du Musée instrumental du Conservatoire royal de musique de Bruxelles, Bruxelles, Les amis de la musique, 5 vol.

MARCUS, Sibyl (1975): Musical Instruments: A Comprehensive Dictionary, New York, The Northern Library.

MATTHYS, Marie-Paule (1986-1987): Traduction et glossaire $(N-F)$ : Bettenhaussen $F$. en Van Krevelen $F$. en $H$. Clavecimbel, clavichord en pianoforte. Stemmen. Stemmingen en onderhoud, mémoire de licence inédit, Provinciale hogeschool voor vertalers en tolken, Gent, 2 vol.

MELLERS, Wilfrid (1970): Textes d'accompagnement de François Couperin, Pièces de clavecin, Ordres $n^{o s}$ $V I I I, X I, X I I I, X N$, [par Rafael Puyana], Disque Philips, coll. «Living Baroque»6770013, s.p.

MERCIER, Philippe (dir.) (1980) : Actes du Colloque international de Louvain, 1976 : La facture de clavecin $d u X V^{e}$ au XVIII siècle, Louvain-la-Neuve, Institut supérieur d'archéologie et d'histoire de l'art, «Publications d'histoire de l'art et d'archéologie de l'Université catholique de Louvain - XXIII», Musicologica neolovaniensia, studia 1, $231 \mathrm{p}$.

MERCIER-YTHIER, Claude (1985) : «À propos des clavecins "Hemsch"» Musique ancienne, $\mathrm{n}^{\circ} 20$, décembre 1985, Bourg-la-Reine, Cael, pp. 82-94.

MERCIER-YTHIER, Claude (1990): Les clavecins, Paris, Éditions Vecteurs, 263 p.

The New Encyclopaedia Britannica in 30 volumes (1977): Micropaedia, 15 th edition, Toronto.

SADIE, Stanley (Ed.) (1984): The New Grove Dictionary of Musical Instruments, London, Macmillan, 3 vol.

SADIE, Stanley (1980): The New Grove Dictionary of Music and Musicians, London, Macmillan, volume 8.

OUELLET, Gaston (1974): Le clavecin: Hier et aujourd'hui, Université de Montréal, thèse, Faculté de musique, 3 vol.

Oxford Mlistrated Dictionary (1965): Toronto, Oxford University Press.

PAUL, John (1981): Modern Harpsichord Makers ; Portraits of Nineteen British Craftsmen and their Work, London, Gollancz, $280 \mathrm{p}$.

PERNON, Gérard (1984) : Dictionnaire de la musique, Paris, Ouest-France, 480 p.

POIRIER, Réjean (1990) : Clavecins à tempéraments; cuvres de Picchi, Bull, Byrd, Kuhnau, Fischer, Dornel, $B a c h$, textes d'accompagnement du disque UMMUS, UMM302.

La Presse (1991) : journal quotidien, Montréal, La Presse, 23 février 1991.

PRO MUSICA (1980) : Recueil de textes de Kenneth Gilbert distribués aux abonnés de la saciété Pro Musica, Montréal, Pro Musica, s.p.

RADIO-CANADA (1991): Services linguistiques, Fichier terminologique Édibase, base DUBUC.

ROBERT, Paul (1987): Dictionnaire alphabétique et analogique de la langue française, Paris, Société du nouveau Littré.

ROBERT, Paul (1985): Dictionnaire alphabétique et analogique de la langue française, $2^{\mathrm{e}}$ édition entièrement revue et enrichie par Alain Rey, Paris, Le Robert, 9 vol. 
RUSSELL, Raymond (1973): The Harpsichord and Clavichord; an Introductory Study, 2nd edition, revised by Howard Schott, London, Faber, 208 p., 103 pl.

RUSSELL, Raymond (1976): «The Harpsichord, Spinet and Virginal», Anthony Baines (dir.), Musical Instruments Through the Ages, New York, Walker and Co., 344 p., pp. 71-83.

SAMOYAULT-VERLET, Colombe (1966): Les facteurs de clavecins parisiens (1550-1793), Paris, Société française de musicologie, Heugel et Cie, 189 p.

SCHAEFFNER, André (1921-1931): «Le clavecin», Encyclopédie de la musique et dictionnaire du Conservatoire, Paris, Delagrave, $2^{\mathrm{e}}$ partie, vol. 3, pp. 2036-2060.

SCHOLES, Percy A. (1970): The Oxford Companion to Music, 10th ed. revised and reset, London, Oxford Press University, $1189 \mathrm{p}$.

THORNTON, Peter (1982): Musical Instruments as Works of Art, 2nd ed., London, England, Victoria and Albert Museum, HMSO, $52 \mathrm{p}$.

TOURNAY, Jean (1987): Archives Dulcken, vol. 1, Bruxelles, The Brussels Museum of Musical Instruments, Schneider Verlag, «Bulletin», vol. XVII, $234 \mathrm{p}$

TOURNAY, Jean (1991): Ce que Jean Tournay construit pour vous, Noville-les-Bois, Belgique, 4 feuillets.

TOURNAY, Jean (1991): Esthétique du clavecin, Noville-les-Bois, Belgique, document inédit.

TOURNAY, Jean (1991): Portrait d'Albertus DELIN suivi de Les Allemands d'Anvers, manuscrit de l'auteur, Noville-les-Bois, Belgique, 44 p. +12 p.

TRANCHEFORT, François-René (1980): Les instruments de musique dans le monde, Paris, Éditions du Seuil, 2 tomes.

VIGNAL, Marc (dir.) (1987): Dictionnaire de la musique, Paris, Larousse, $882 \mathrm{p.}$

Webster Third International Dictionary of the English Language (1981): Unabridged, Springfield MA, Merriam-Webster Inc.

WHITFIELD, Charles (1989) : L'anglais musicologique ; l'anglais des musiciens, Paris, Beauchesne, 149 p.

ZUCHERMANN, Wolfgang Joachim (1969): The Modern Harpsichord; Twentieth-Century Instruments and Their Makers, London, Peter Owen, 255 p. 